\title{
On the Reduction of an Arbitrary Real Square Matrix to Tridiagonal Form
}

\author{
By H. H. Wang and R. T. Gregory
}

1. This note is written for the purpose of making some remarks relative to a paper by C. D. La Budde [1] in which he describes an algorithm for tridiagonalizing an arbitrary real square matrix using similarity transformations. We shall refer to equations in [1] by their numbers.

2. La Budde's algorithm is composed of $n-2$ steps. The generic $j$ th step consists essentially of a similarity transformation of the form (see (3.4) in [1])

$$
A^{\prime}=\left[V_{j}\left(I_{n-j}+a X Y^{T}\right)\right] A\left[V_{j}\left(I_{n-j}+b X Y^{T}\right)\right],
$$

where the components of $X$ and $Y$ (see (3.8)-(3.11) in [1]) are chosen so as to produce zeros in the off-tridiagonal positions of the $j$ th row and $j$ th column of $A^{\prime}$. Thus at the completion of the $(n-2)$ th step the matrix is in tridiagonal form. A sufficient condition for continuation at the $j$ th step is that the number $S_{j}$ (see (2.5) in [1]) does not vanish.

3. La Budde [1, p. 436] says, "In order to continue the algorithm into step $j+1$, we must be certain that $S_{j+1}^{\prime} \neq 0 \ldots$ Now $a, b$ are arbitrary (except for sign) so we may theoretically choose $|a|,|b|$ so that $S_{j+1} \neq 0 \ldots .|a|,|b|$ could be determined by trial and error starting ...." It is our purpose to demonstrate that we may not always be able to choose $|a|$ and $|b|$ in order to insure that $S_{j+1}^{\prime} \neq 0$. We shall do this by displaying some matrices which are counter examples. These matrices fall into two categories.

First category. If at some step of the reduction, say the $i$ th step, the matrix $A$ is in the "reduced form" (here and afterwards the subscripts denote the size of the submatrix)

$$
\left[\begin{array}{c:c}
A_{t} & A_{t, n-t} \\
\hdashline 0 & A_{n-t}
\end{array}\right]
$$

with $t \geqq i$, where

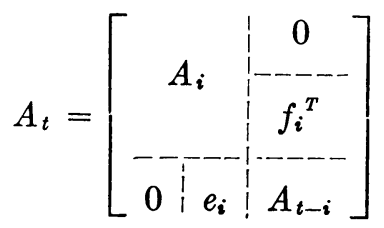

and where $A_{i}$ is an $i \times i$ tridiagonal matrix and $f_{i}$ and $e_{i}$ are vectors of dimension $t-i$, then, not later than step $t$, the procedure will fail to continue. This will happen because at the $(t-1)$ th step the matrix $A$ will have the form

Received November 19, 1963. Revised January 24, 1964. Work on this paper was supported in part by the National Science Foundation under Grant GP-217 and by the U. S. Army Research Office (Durham) under Grant DA-ARO(D)-31-124-G388. 


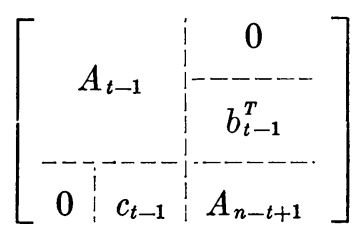

in which $c_{t-1}$ is a null vector except for its first component $a_{t, t-1}$, and the first column of $A_{n-t+1}$ is null except for its first element $a_{t t}$. Hence, no matter what values of $a$ and $b$ are chosen, the vector $X$ will have its last $(n-t)$ components zero. Therefore, in the matrix $A^{\prime}$ of (1),

$$
a_{k t}^{\prime}=0, \quad(k=t+1, \cdots, n) .
$$

Consequently, $S_{t}^{\prime}=0$ and the procedure cannot be continued.

At this point we give two numerical examples to illustrate the failure of the algorithm when the matrix is in reduced form. In the first example the reduced form emerges at the second step. In the second example the original matrix is in reduced form.

Example 1. The original matrix is

$$
\left[\begin{array}{rrrrr}
4 & 1 & 0 & 0 & 0 \\
1 & 4 & 0 & -3 & 0 \\
2 & 0 & 5 & 1 & 1 \\
-2 & 2 & 0 & 4 & 0 \\
4 & 1 & -1 & 6 & 2
\end{array}\right]
$$

During the first step suppose we choose $a=b=-2$. In this case

$$
\begin{aligned}
S_{1} & =1, \\
1 / c & =1, \\
X^{T} & =(1,1,-1,2), \\
Y^{T} & =(1,0,0,0),
\end{aligned}
$$

and at the completion of the first step the matrix becomes

$$
\left[\begin{array}{rrrrr}
4 & -1 & 0 & 0 & 0 \\
-1 & 10 & 0 & 3 & 0 \\
0 & 8 & 5 & 7 & 1 \\
0 & -14 & 0 & -2 & 0 \\
0 & 60 & 1 & 18 & 2
\end{array}\right] .
$$

Before beginning the second step we notice that $S_{2} \neq 0$ and $a_{24} \neq 0$ but $a_{23}=0$. Therefore, a permutation transformation $P_{34}$ (see (2.2) in [1]) must be performed before we can carry out the second step. However, after the permutation the matrix is

$$
\left[\begin{array}{rrr:rc}
4 & -1 & 0 & 0 & 0 \\
-1 & 10 & 3 & 0 & 0 \\
0 & -14 & -2 & 0 & 0 \\
\hdashline 0 & 8 & 7 & 5 & 1 \\
0 & 60 & 18 & 1 & 2
\end{array}\right]
$$


which is in reduced form. At this stage no matter what values we choose for $a$ and $b, Y$ will always have its last two components zero. Hence, $a_{34}^{\prime}$ and $a_{35}^{\prime}$ (and therefore $S_{3}{ }^{\prime}$ ) always equal zero, and the procedure will break down at the third step.

Example 2. The original matrix is

$$
\left[\begin{array}{rrr:rr}
2 & 1 & 0 & -2 & 2 \\
1 & 3 & 1 & 2 & 3 \\
2 & 7 & 2 & 1 & -1 \\
\hdashline 0 & 0 & 0 & 4 & 1 \\
0 & 0 & 0 & 1 & 3
\end{array}\right] .
$$

During the first step suppose we choose $a=b=-2$. Consequently,

$$
\begin{aligned}
S_{1} & =1, \\
1 / c & =1, \\
X^{T} & =(1,1,0,0), \\
Y^{T} & =(1,0,-1,1) .
\end{aligned}
$$

At the end of the first step the matrix becomes

$$
\left[\begin{array}{rrrrr}
2 & -1 & 0 & 0 & 0 \\
-1 & 5 & -1 & -4 & 10 \\
0 & -1 & 0 & -1 & -9 \\
0 & 0 & 0 & 4 & 1 \\
0 & 0 & 0 & 1 & 3
\end{array}\right]
$$

In this matrix both $S_{2} \neq 0$ and $a_{32}=a_{23} \neq 0$. Therefore, we can carry out the second step. However, at this stage, regardless of the values we choose for $a$ and $b, X$ will always have its last two components zero. Therefore, we always have $S_{3}{ }^{\prime}=0$, and the procedure will break down at the third step.

Second category. If at some step $i$, the matrix has the form

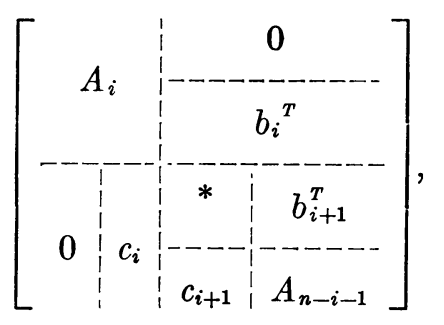

where $A_{i}$ is a tridiagonal matrix of order $i$, where $c_{i}{ }^{T}$ and $b_{i}{ }^{T}$ have the form

$$
(q, 0,0, \cdots, 0)
$$

with $q \neq 0$, and where

$$
S_{i+1}=c_{i+1}^{T} b_{i+1}=0
$$

with neither of the $(n-i-1)$ dimensional vectors $c_{i+1}$ or $b_{i+1}$ vanishing, then it is quite clear that the procedure breaks down at the $(i+1)$ th step. 
Example 3. The matrix

$$
\left[\begin{array}{cc:ccc}
4 & 2 & 0 & 0 & 0 \\
& & 0 & 0 \\
1 & 4 & 2 & 0 & 0 \\
\hdashline 0 & 1 & 1 & 2 & 0 \\
& & & 0 & 2 \\
0 & 0 & 0 & 2 & 1 \\
0 & 0 & 2 & 1 & 3
\end{array}\right]
$$

is a matrix of this type.

\section{Remedies.}

(a) If the matrix is in the reduced form

$$
\left[\begin{array}{c:c}
A_{i} & A_{i, n-i} \\
\hdashline 0 & A_{n-i}
\end{array}\right]
$$

we can always replace the problem by the simpler problem of tridiagonalizing $A_{i}$ and $A_{n-i}$. Of course this will change La Budde's statement $[1$, p. 437], " . . the characteristic polynomial of the matrix $A$ will not factor under this algorithm."

(b) Upon discovering that we have applied the procedure to a matrix of the second category we must begin anew after some "preconditioning" of the matrix. For example, this preconditioning can be carried out by a $T_{k 1}(a)$ transformation (see (2.1) in [1]) or by a $T_{1 j}(b)$ transformation or even by a series of $T_{k 1}(a)$ and $T_{1 j}(b)$ transformations, where in all cases $1<j, k \leqq n$. However, we do not know beforehand how the matrix must be preconditioned in order that the tridiagonalization can be carried out to completion. Hence, the preconditioning is on a trial and error basis.

To illustrate, consider (8) above. We precondition the matrix by a $T_{31}(1)$ transformation, i.e.,

$\left[\begin{array}{lllll}1 & 0 & 0 & 0 & 0 \\ 0 & 1 & 0 & 0 & 0 \\ 1 & 0 & 1 & 0 & 0 \\ 0 & 0 & 0 & 1 & 0 \\ 0 & 0 & 0 & 0 & 1\end{array}\right]\left[\begin{array}{lllll}4 & 2 & 0 & 0 & 0 \\ 1 & 4 & 2 & 0 & 0 \\ 0 & 1 & 1 & 2 & 0 \\ 0 & 0 & 0 & 2 & 1 \\ 0 & 0 & 2 & 1 & 3\end{array}\right]\left[\begin{array}{lllll}1 & 0 & 0 & 0 & 0 \\ 0 & 1 & 0 & 0 & 0 \\ -1 & 0 & 1 & 0 & 0 \\ 0 & 0 & 0 & 1 & 0 \\ 0 & 0 & 0 & 0 & 1\end{array}\right]=\left[\begin{array}{rllll}4 & 2 & 0 & 0 & 0 \\ -1 & 4 & 2 & 0 & 0 \\ 3 & 3 & 1 & 2 & 0 \\ 0 & 0 & 0 & 2 & 1 \\ -2 & 0 & 2 & 1 & 3\end{array}\right]$.

If we choose $a=-b=2$, then the transformation for the first step becomes

$$
\begin{array}{r}
{\left[\begin{array}{rrrrr}
1 & 0 & 0 & 0 & 0 \\
0 & 1 & 0 & 0 & 0 \\
0 & 3 & 1 & 0 & 0 \\
0 & 0 & 0 & 1 & 0 \\
0 & -2 & 0 & 0 & 1
\end{array}\right]\left[\begin{array}{rrrrr}
4 & 2 & 0 & 0 & 0 \\
-1 & 4 & 2 & 0 & 0 \\
3 & 3 & 1 & 2 & 0 \\
0 & 0 & 0 & 2 & 1 \\
-2 & 0 & 2 & 1 & 3
\end{array}\right]\left[\begin{array}{rrrrr}
1 & 0 & 0 & 0 & 0 \\
0 & 1 & 0 & 0 & 0 \\
0 & -3 & 1 & 0 & 0 \\
0 & 0 & 0 & 1 & 0 \\
0 & 2 & 0 & 0 & 1
\end{array}\right]} \\
=\left[\begin{array}{rrrrr}
4 & 2 & 0 & 0 & 0 \\
-1 & -2 & 2 & 0 & 0 \\
0 & -6 & 7 & 2 & 0 \\
0 & 2 & 0 & 2 & 1 \\
0 & 4 & -2 & 1 & 3
\end{array}\right] .
\end{array}
$$


If we again choose $a=-b=2$, the second transformation becomes

$$
\begin{array}{r}
{\left[\begin{array}{lllll}
1 & 0 & 0 & 0 & 0 \\
0 & 1 & 0 & 0 & 0 \\
0 & 0 & 1 & 0 & 0 \\
0 & 0 & \frac{1}{3} & 1 & 0 \\
0 & 0 & \frac{2}{3} & 0 & 1
\end{array}\right]\left[\begin{array}{rrrrr}
4 & 2 & 0 & 0 & 0 \\
-1 & -2 & 2 & 0 & 0 \\
0 & -6 & 7 & 2 & 0 \\
0 & 2 & 0 & 2 & 1 \\
0 & 4 & -2 & 1 & 3
\end{array}\right]\left[\begin{array}{rrrrr}
1 & 0 & 0 & 0 & 0 \\
0 & 1 & 0 & 0 & 0 \\
0 & 0 & 1 & 0 & 0 \\
0 & 0 & -\frac{1}{3} & 1 & 0 \\
0 & 0 & -\frac{2}{3} & 0 & 1
\end{array}\right]} \\
=\left[\begin{array}{rrrrr}
4 & 2 & 0 & 0 & 0 \\
-1 & -2 & 2 & 0 & 0 \\
0 & -6 & \frac{19}{3} & 2 & 0 \\
0 & 0 & \frac{7}{9} & \frac{8}{3} & 1 \\
0 & 0 & -\frac{1}{9} & \frac{7}{3} & 3
\end{array}\right] .
\end{array}
$$

In the matrix on the right we see that

$$
S_{3} \neq 0
$$

and we can carry out the third step. At the completion of the third step, the matrix will be in tridiagonal form.

The University of Texas

Austin, Texas

1. C. D. LA BUDDE, "The reduction of an arbitrary real square matrix to tri-diagonal form using similarity transformations," Math. Comp., v. 17, 1963, p. 433-437.

\section{A Note on La Budde's Algorithm}

\section{By Beresford Parlett}

In the October 1963 issue of Mathematics of Computation, La Budde presented an algorithm for the reduction of an arbitrary real square matrix $A$ to a similar tridiagonal matrix. We show here that when applied to Hessenberg matrices this procedure is identical to the more familiar reduction by elimination methods. Therefore the same care is needed with the new technique as with elimination in treating the instabilities which can occur, see [1] and [3].

Let $A$ be an unreduced lower Hessenberg matrix; i.e., $a_{i j}=0$ if $j>i+1$, $a_{i, i+1} \neq 0$. La Budde's algorithm [2] consists of a sequence of major steps at the $j$ th of which the current matrix $A$ is transformed to

$$
A^{\prime}=V_{j}\left(I_{n-j}+a x y^{t}\right) A V_{j}\left(I_{n-j}+b x y^{t}\right)
$$

using the notation of [2]. The equations (3.8)-(3.11) determining the vectors $x, y$ and the scalar $c^{-1}=\sum_{k=j+1}^{n} x_{k} a_{j k}$ reduce, in this case, to

$$
c^{-1}=-(a+b) a_{j, j+1} a_{j+1, j} / a b
$$

Received December 5, 1963. The work presented in this paper is supported by the AEC Computing and Applied Mathematics Center, Courant Institute of Mathematical Sciences, New York University, under Contract AT(30-1)-1480 with the U. S. Atomic Energy Commission. 